Z Rheumatol 2020 · 79:967-968

https://doi.org/10.1007/s00393-020-00908-x

Angenommen: 3. August 2020

(c) Springer Medizin Verlag $\mathrm{GmbH}$, ein Teil von Springer Nature 2020

\begin{abstract}
„Die Wissenschaft ist Teil der Lebenswirklichkeit; sie ist das Was, das Wie und das Warum von allem in unserer Erfahrung." (Rachel Carson, Biologin, In Paul Brooks The House of Life)
\end{abstract}

Liebe Kolleginnen und Kollegen,

die Versorgungsforschung widmet sich Fragen des Zugangs zur Gesundheitsversorgung, der Effektivität und Effizienz von Versorgungsangeboten sowie deren Qualität mit dem Ziel, die Zugangs-, Verteilungs- und Bedarfsgerechtigkeit im Versorgungsalltag zu erhöhen. Sie fragt nach dem Was, Wie und Warum in der Versorgung, um Letztere für die Patienten zu verbessern. Lange Zeit war sie als vierter Pfeiler der Gesundheitsforschung neben der Grundlagenforschung, der krankheitsorientierten Forschung im Labor und der klinischen Forschung unterschätzt. Erst nach der Jahrtausendwende ist sie hierzulande $\mathrm{zu}$ einer bedeutsamen und von vielen Arbeitsgruppen verfolgten inter- und transdisziplinären Forschungsrichtung geworden, auf die zahlreiche Förderinitiativen des Bundesministeriums für Bildung und Forschung, des Bundesministeriums für Gesundheit (BMG) und des Innovationsfonds des Gemeinsamen Bundesausschusses abzielen.

Sehr viel früher war man sich in der Rheumatologie der Notwendigkeit versorgungsbezogener Forschung bewusst. Bereits zu Beginn der 1990er-Jahre wurde im Rahmen der BMG-Förderung zum Aufbau der regionalen kooperativen Rheumazentren die Kerndokumenta-

\author{
Kirsten Minden ${ }^{1,2}$. Andreas Radbruch ${ }^{1}$ Gerd-Rüdiger Burmester ${ }^{2}$. \\ Anja Strangfeld ${ }^{1}$ \\ 'Deutsches Rheuma-Forschungszentrum Berlin, Berlin, Deutschland \\ ${ }^{2}$ Medizinische Klinik mit Schwerpunkt Rheumatologie und Klinische Immunologie, Charité - \\ Universitätsmedizin Berlin, Berlin, Deutschland
}

\title{
Das Was, Wie und Warum in der rheumatologischen Versorgung
}

tion zur evaluativen Begleitforschung auf den Weg gebracht. Mit ihr werden seitdem Strukturen, Prozesse und Ergebnisse rheumatologischer Versorgung abgebildet, analysiert und Versorgungsdefizite transparent gemacht. Aber auch Verbesserungen in der wohnortnahen Versorgung von Rheumakranken lassen sich darstellen.

Sowohl die Kerndokumentation als auch das Aufgabengebiet der regionalen kooperativen Rheumazentren wurden in den über 25 Jahren seit ihrer Initiierung weiterentwickelt, was Barbara Gundelach und Christof Specker in ihrem Beitrag eindrucksvoll vor Augen führen. Als Arbeitsgemeinschaft Regionaler Kooperativer Rheumazentren in der Deutschen Gesellschaft für Rheumatologie (DGRh) tragen sie heute mit Kampagnen und vielen Aktivitäten zur Sichtbarkeit des Fachgebietes und auf wissenschaftlicher Seite zur Verbesserung der rheumatologischen Versorgungsforschung in Deutschland bei.

Julia Rautenstrauch hat die Kerndokumentation im Rahmen des „Kompetenznetzes Rheuma“ von 1999 bis 2007 und der nachfolgenden Unterstützung durch die Arbeitsgemeinschaft der Regionalen Kooperativen Rheumazentren sowie die korporativen Mitglieder der DGRh als Pressereferentin und später als Generalsekretärin der DGRh begleitet. Sie verweist in ihrem Beitrag darauf, dass die im Rahmen der Kerndokumentation gewonnenen Erkenntnisse bis heute eine der wichtigsten Datenquellen für die Entwicklung von Leitlinien und Empfehlungen in der deutschen Rheumatologie bilden. Verstärkt wird diese Datenbasis durch weitere, in den letzten Jahren im Programmbereich Epidemiologie und Versorgungsforschung am Deutschen Rheuma-Forschungszentrum in Berlin (DRFZ) aufgebaute große Patientenkohorten.

Einen Überblick über die am DRFZ durchgeführten Beobachtungsstudien gibt der Beitrag von Yvette Meißner und Koautoren. Derzeit werden hier in $10 \mathrm{Be}$ obachtungsstudien über 30.000 Patienten aller Altersgruppen mit rheumatischen Erkrankungen erfasst und regelmäßig $\mathrm{zu}$ ihrer Lebenssituation und -qualität befragt. Ärzte werden um Auskunft zur Therapie, zum Gesundheitszustand und zur Aktivität der rheumatischen Erkrankung der von ihnen behandelten Patienten gebeten. So können Verlauf und Konsequenzen rheumatischer Erkrankungen und der Einfluss der Behandlung auf das Befinden der Patienten untersucht werden. Im Mittelpunkt der Forschung stehen die Menschen mit rheumatischen und muskuloskelettalen Erkrankungen; oberstes Ziel ist die Verbesserung ihrer Lebenssituation.

Rotraut Schmale-Grede und Gisela Faubel führen in ihrem Beitrag aus, wie wertvoll die Daten aus der rheumatologischen Versorgungsforschung auf verschiedenen Ebenen für die Betroffenen selbst und die Arbeit der Deutschen Rheuma-Liga (DRL) sind. Nachgewiesene Versorgungsdisparitäten, aufgezeigte Krankheitslast, aber auch Erfolge in der medizinischen Betreuung gestatten es unter anderem, wissenschaftsbasiert gesundheitspolitischen Forderungen mehr Nachdruck zu verleihen. 
Die Grundlagen für die rheumatologische Versorgungsforschung am DRFZ hat Professor Dr. rer. pol. Angela Zink geschaffen, der dieser Schwerpunkt gewidmet ist. Angela Zink hat im Jahr 1991 mit dem Aufbau der Kerndokumentation und des Programmbereichs Epidemiologie und Versorgungsforschung am DRFZ begonnen, diesen Bereich geleitet und inhaltlich ausgerichtet. Von anfänglich 4 Mitarbeitern wuchs der Bereich stetig mit den Forschungsvorhaben auf ein multidisziplinäres Team von inzwischen mehr als 60 Ärzten, Statistikern, Gesundheitswissenschaftlern, medizinischen Dokumentaren, Studenten und anderen wissenschaftlichen Mitarbeitern an. Dass die bundesweiten Beobachtungsstudien des DRFZ eine derart große Akzeptanz finden und in enger Zusammenarbeit mit über 400 pädiatrischen, internistischen, ophthalmologischen, orthopädischen und hausärztlichen Einrichtungen aller Versorgungsstufen durchgeführt werden, ist Angela Zink und ihrem unermüdlichen Werben und Engagement für eine bestmögliche rheumatologischepidemiologische Datenbasis zu verdanken. Sie hat die epidemiologische Forschung und die Versorgungsforschung auf dem Gebiet der Rheumatologie national und international entscheidend vorangebracht. Über 170 wissenschaftliche Veröffentlichungen und zahlreiche Buchbeiträge zeugen davon. Ihr Einsatz und wissenschaftliches Euvre wurden u. a. mit dem Bundesverdienstkreuz, der Carol-Nachman-Medaille, der Francois-Blondel-Medaille und dem RheoFortschrittspreis gewürdigt.

》) Die Daten aus der rheumato-
logischen Versorgungsforschung
sind für Betroffene sowie DRL
sehr wertvoll

Die von ihr geschaffenen Strukturen, die methodische Expertise und das von ihr geknüpfte nationale und internationale Netzwerk gilt es jetzt zu nutzen, um die Herausforderungen der kommenden Jahre mit der zunehmenden Digitalisierung und der Verfügbarkeit von sehr großen und vielfältigen Datenmengen zu meistern. Bereits in den vergangenen Jahren wurde mit der zunehmenden Digitalisierung der laufenden Forschungsvorhaben und der Integration neuer Datenquellen die Grundlage für eine erfolgreiche Fortführung und Weiterentwicklung der Forschungsaktivitäten geschaffen, wie Katinka Albrecht und Kollegen darstellen. Apps und Tablets zur Datenerfassung werden genutzt, interaktive Apps wie eine Medikamenten-App im RABBIT-SpA-Register und eine motivierende App für junge Rheumatiker kommen gerade dazu. Aktivitätsmonitore werden in den nächsten Monaten umfassende Daten zu Aktivitätsprofilen von Patienten liefern. Durch den Zugang zu Daten der Barmer Krankenkasse wird sich die Versorgung auf Bevölkerungsebene noch umfangreicher darstellen lassen. Mit der Sammlung von Bioproben in fast allen Patientenkohorten ist ein strategisch entscheidender Schritt vollzogen worden, damit epidemiologische Daten (z.B. definierte Phänotypen) zusammen mit genetischen und immunologischen Daten erfasst werden und der Umsetzung einer stratifizierten bzw. individualisierten Therapie dienen können.

Im März 2020 hat Angela Zink die Leitung des Programmbereiches am DRFZ nach ihrer Pensionierung abgegeben. Für das, was sie geschaffen, ermöglicht und auf den Weg gebracht hat, sind ihr die Mitarbeiter des DRFZ, Wissenschaftler aus dem In- und Ausland, unzählige Kliniker und Patienten mit rheumatischen Erkrankungen außerordentlich dankbar. Ziel des Programmbereichs Epidemiologie und Versorgungsforschung am DRFZ ist eine klinisch relevante Forschung. Den von Angela Zink vorgezeichneten Weg weiterzugehen und dabei neue Möglichkeiten $\mathrm{zu}$ integrieren ist Ansporn und Herausforderung zugleich.
Wir wünschen Angela Zink privat und für ihre weitere wissenschaftliche Tätigkeit alles erdenklich Gute und den Lesern dieser Ausgabe der Zeitschrift für Rheumatologie viel Freude beim Lesen.

Ihre/Ihr

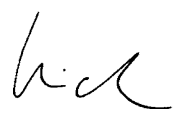

Kirsten Minden

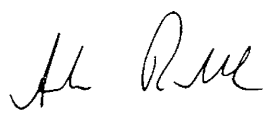

Andreas Radbruch

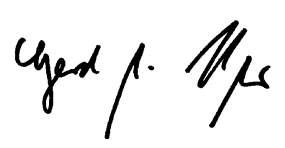

Gerd-Rüdiger Burmester

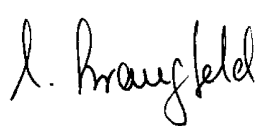

Anja Strangfeld

\section{Korrespondenzadresse}

Prof. Dr. Kirsten Minden

Deutsches Rheuma-Forschungszentrum Berlin Charitéplatz 1, 10117 Berlin, Deutschland minden@drfz.de

\section{Prof. Dr. Andreas Radbruch}

Deutsches Rheuma-Forschungszentrum Berlin Charitéplatz 1, 10117 Berlin, Deutschland radbruch@drfz.de

\section{Prof. Dr. Gerd-Rüdiger Burmester}

Medizinische Klinik mit Schwerpunkt

Rheumatologie und Klinische Immunologie,

Charité - Universitätsmedizin Berlin

Charitéplatz 1, 10117 Berlin, Deutschland

gerd.burmester@charite.de

\section{PD Dr. Anja Strangfeld}

Deutsches Rheuma-Forschungszentrum Berlin Charitéplatz 1, 10117 Berlin, Deutschland strangfeld@drfz.de

Interessenkonflikt. K. Minden, A. Radbruch G.-R. Burmester und A. Strangfeld geben an, dass kein Interessenkonflikt besteht. 